\title{
Polarized surface-enhanced Raman spectroscopy of suspended carbon nanotubes by Pt-Re nanoantennas
}

\author{
Christian Bäuml, Tobias Korn, Christoph Lange, Christian Schüller, Christoph Strunk, and Nicola Paradiso* \\ Institut für Experimentelle und Angewandte Physik, University of Regensburg, 93053 Regensburg, Germany
}

(Received 17 March 2017; published 7 July 2017)

\begin{abstract}
We present optical nanoantennas designed for applications that require processing temperatures larger than $800^{\circ} \mathrm{C}$. The antennas consist of arrays of Re/Pt bilayer strips fabricated with a lift-off-free technique on top of etched trenches. Reflectance measurements show a clear plasmonic resonance at approximately $670 \mathrm{~nm}$ for light polarized orthogonal to the strip axis. The functionality of the antennas is demonstrated by growing single-walled carbon nanotubes (CNTs) on top of the antenna arrays and measuring the corresponding Raman signal enhancement of selected CNTs. The results of the measurements are quantitatively discussed in light of numerical simulations which highlight the impact of the substrate.
\end{abstract}

DOI: 10.1103/PhysRevB.96.035408

The key feature of plasmonic nanostructures is their ability to confine light on a much smaller scale compared to the far field wavelength [1,2]. As a consequence, such structures are able to amplify the optical near field at their surface by many orders of magnitude, especially if a localized surface plasmon resonance (LSPR) is excited. This has a dramatic impact on optical spectroscopy, where the near field amplification makes it possible to detect spectra of single molecules [3-5]. In particular, the use of plasmonic structures represented a fundamental breakthrough for Raman spectroscopy, where it is referred to as surface-enhanced Raman spectroscopy (SERS) [6-8]. The name reflects the fact that early experiments made use of granular metallic surfaces $[6,9,10]$. Subsequently, colloidal $\mathrm{Au}$ or Ag nanoparticles [10-12], as well as periodically indented metal layers [13,14], were used as plasmonic resonators. Such resonators act as optical nanoantennas, i.e., they concentrate propagating radiation into a subwavelength-sized region where the near field dominates the far field.

The progress of nanoplasmonics enabled the fabrication of optical nanoantennas specifically designed to work in combination with given target molecules $[3,4]$. The combination of plasmonic structures with specific devices has nevertheless a limit: the fabrication of the target device or the synthesis of the desired molecule is not always compatible with the fabrication of the optical nanoantennas. Carbon nanotubes (CNTs) represent a good example of such a situation. The fabrication of ultraclean devices [15] often requires that the CNT growth must be performed as a last fabrication step $[16,17]$. CNTs are grown by chemical vapor deposition (CVD) at temperatures of the order of $800^{\circ} \mathrm{C}$ or higher [18]. These temperatures are sufficient to melt thin films or nanoparticles of the most common plasmonic materials, namely Au and Ag. Therefore, so far the proposed prototypes [19-22] of optical nanoantennas for CNTs had to be fabricated or deposited after the CNT growth, i.e., they were not compatible with the ultraclean fabrication scheme.

The low degree of disorder in ultraclean CNTs allows for a detailed comparison of quantum transport experiments with microscopic theories [17]. For such analysis an independent

*nicola.paradiso@physik.uni-regensburg.de determination of diameter and chiral angle is highly desirable $[23,24]$. Such information could in principle be provided by Raman spectroscopy, but only in the rare case that the energy of the incident or scattered photon energy matches the energy separation $E_{i i}$ between two van Hove singularities (VHS) of the CNT density of states [25-27].

In experiments with CNTs grown on top of predefined electrodes one deals typically with few devices. Without the usage of tunable lasers it is unlikely to meet the Raman resonance condition for an individual contacted CNT. The field amplification induced by plasmonic structures can be crucial to obtain a sizable optical signal from a limited number of contacted devices.

In the present work we developed optical nanoantennas which are resistant to the extreme conditions of the nanotube CVD growth. We use spatially resolved reflectance measurements to demonstrate the occurrence of a localized surface plasmon resonance (LSPR) at around 630-670 nm. Finally, we exploit the induced field enhancement to magnify the Raman signal of individual ultraclean CNTs.

\section{EXPERIMENTAL DETAILS}

Our sample fabrication is sketched in Fig. 1. The antenna arrays are fabricated on degenerately doped silicon substrates with a $470 \pm 10$-nm-thick $\mathrm{SiO}_{2}$ cap layer. The fabrication process starts with the etching of trenches defined by electron beam lithography (EBL). 30-nm-wide, 100-nm-deep, and $6-\mu \mathrm{m}$-long trenches are distributed in arrays of nine elements with a periodicity of $240 \mathrm{~nm}$. Four arrays are arranged as the four sides of a rectangle. On top of the trenches, we deposited a $\mathrm{Re}-\mathrm{Pt}$ (with thickness 7 and $18 \mathrm{~nm}$, respectively) metal bilayer. The choice of Re as sticking layer is motivated by its extraordinary stability at high temperatures. Even when reduced to a few-nanometer-thin layer, Re can stand temperatures as high as $900{ }^{\circ} \mathrm{C}$ with negligible structural deformation. On the other hand, at these temperatures $\mathrm{Pt}$ nanostructures suffer major roughening, while most metals (including common plasmonic materials as, e.g., $\mathrm{Au}, \mathrm{Ag}, \mathrm{Pd}, \mathrm{Al}$ ) simply melt. We found that the combination of Re as sticking layer and Pt as top layer is the best compromise between the thermal stability provided 

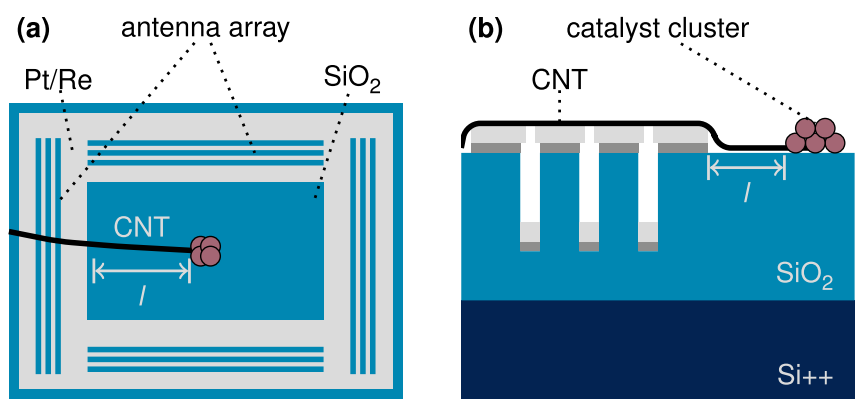

(c) After CVD step at $850^{\circ} \mathrm{C}$

(d)

Zoom-in

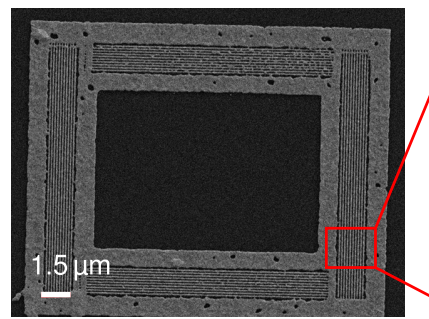

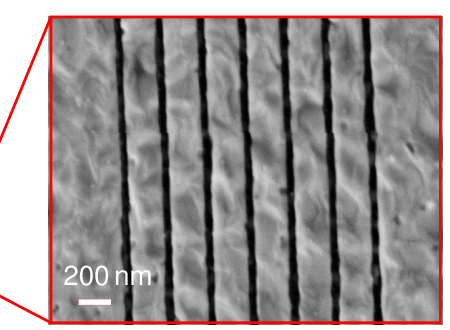

FIG. 1. (a) Sketch of the sample in top view. (b) Side-view scheme. Carbon nanotubes (CNTs) start growing from clusters of catalyst particles (brown circles) deposited in the middle of the rectangular structure. The antenna structures are defined by evaporating a Re-Pt bilayer on top of an array of etched trenches. (c) Scanning electron microscope (SEM) micrograph of a structure after the chemical vapor deposition $(\mathrm{CVD})$ step at $850^{\circ} \mathrm{C}$. (d) Zoom in on the right antenna array. The effect of the high temperature of the CVD step is limited to a modest $(\approx 5 \mathrm{~nm})$ corrugation of the metal surface.

by Re, and the plasmonic performances and chemical stability guaranteed by Pt.

Etched trenches make possible to control the gap width between adjacent metal strips: the thicker the metal layer, the thinner is the residual gap [28]. The metallization does not cover the entire sample surface, but it is limited to EBL-defined square frames overlapping the trenches, as shown in Figs. 1(a) and 1(c). In particular the inner part of the rectangular frame is not metallized: here a $1-\mu \mathrm{m}$-wide cluster of catalyst nanoparticles is patterned by EBL [29].

The last fabrication step is the CVD growth. This step takes place at $850^{\circ} \mathrm{C}$ under a steady stream of $\mathrm{CH}_{4}(10 \mathrm{sccm})$ and $\mathrm{H}_{2}$ $(20 \mathrm{sccm})$ in an inert atmosphere of argon $(1500 \mathrm{sccm})$ at 1 bar. CNTs start growing vertically from the catalyst cluster. Owing to van der Waals interaction, they bend downwards during the growth until they eventually fall on the substrate or on the antennas [30]. Most CNTs are shorter than the cluster-toantenna distance $l \approx 4 \mu \mathrm{m}$. However, some CNTs grow long enough to bridge the antennas. Owing to the small gap between the antenna strips, the CNTs are suspended over the substrate. The CNT position and orientation is clearly unpredictable, thus it is determined a posteriori by atomic force microscopy (AFM).

Beside the antenna structure described above we have investigated also alternative structures where $\mathrm{Ti}$ is used as sticking layer instead of Re. Although SERS is still possible with such structures, their thermal stability is not comparable to that of $\mathrm{Re} / \mathrm{Pt}$ antennas. Such structures are discussed in detail in the Supplemental Material [29].

Raman and reflectance measurements are performed in a confocal setup [7,22,29]. Light is transmitted through a beam splitter and focused on the sample by a $100 \times$ objective lens. The light spot size is approximately $2 \mu \mathrm{m}$ for white light and below $1 \mu \mathrm{m}$ for the two laser lines, i.e., the spatial resolution is comparable to the array width. The scattered light is transmitted again through the beam splitter and then sent to the detector, as discussed in our previous work [22]. The configuration of light sources, detectors, and polarizers depends on the specific measurement [29]. For reflectance spectroscopy we use a thermal source of white light and a spectrometer as detector. An analyzer is placed in between the sample and the spectrometer. For reflectance maps the light source is a laser (either a He/Ne laser with $\lambda_{L}=633 \mathrm{~nm}$ or a diode laser with $\lambda_{L}=532 \mathrm{~nm}$ ) and the detector is a power meter. A polarizer is placed between the source and the sample. The same configuration is used for Raman spectroscopy. In this case the detector is a spectrometer.

\section{EXPERIMENTAL RESULTS}

Long metal nanowires display a plasmonic resonance near the visible when excited with a field orthogonal to their axis, where the incompressible electron plasma experiences a nonnegligible restoring force $[1,28]$. The excitation of a LSPR implies losses due to absorption in the metal, which results in a reduction of the scattered light intensity.

To demonstrate that the Re/Pt arrays still behave as directional optical antennas even after the CVD step, we acquired maps of the reflected signal as a function of the polarization of the incident light. We use monochromatic light from a laser source (with $\lambda_{L}=633$ or $532 \mathrm{~nm}$ ), focused to a $0.5-\mu \mathrm{m}$-wide spot. Figure 2 shows how reflectance maps evolve when the polarization of the incident light at $\lambda_{L}=633 \mathrm{~nm}$ is gradually

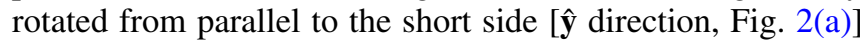
to parallel to the long side [ $\hat{\mathbf{x}}$ direction, Fig. 2(f)] of the rectangular pattern in Fig. 1(c). The maps in Fig. 2 clearly show that antennas directed along $\hat{\mathbf{x}}$ have a minimum in the reflected signal for incident light polarization directed along $\hat{\mathbf{y}}$, and vice versa. This corresponds to the excitation of a LSPR as discussed above. By rotating the incident light polarization the reflected signal from antennas directed along $\hat{\mathbf{x}}$ continuously increases, while the signal from antennas directed along $\hat{\mathbf{y}}$ decreases accordingly. This behavior is not observed for the green laser $\left(\lambda_{L}=532 \mathrm{~nm}\right)$, because this wavelength is far from the LSPR minimum [29]. The LSPR resonance near the $633 \mathrm{~nm}$ can be also observed by reflectance spectroscopy, as reported in the Supplemental Material [29].

Having demonstrated the occurrence of a plasmonic resonance near $\lambda_{L}=633 \mathrm{~nm}$, we used antenna arrays to amplify the Raman signal of selected suspended CNTs. After the CVD growth process, AFM scans are used to locate the presence of individual CNTs and where exactly they cross the antenna arrays. A convenient CNT is in this case one far from other CNTs and long enough to completely bridge the antenna array.

Figure 3 shows the results of Raman measurements performed on three samples using the $\mathrm{He} / \mathrm{Ne}$ laser $(\lambda=633 \mathrm{~nm})$. 
(a)

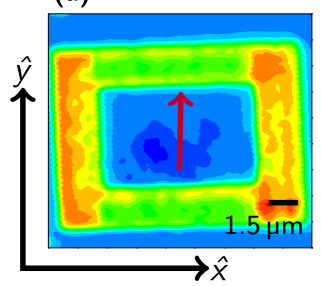

(b)

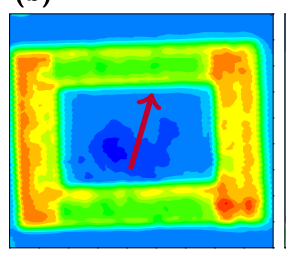

(c)

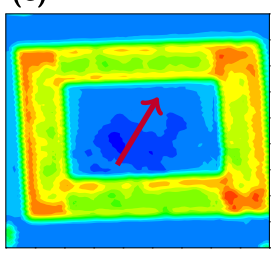

(d)

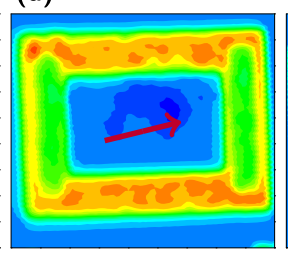

(e)

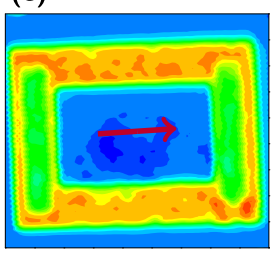

(f)

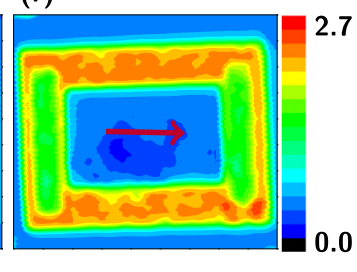

FIG. 2. Reflectance maps measured on Re/Pt antennas for incident wavelength $\lambda_{L}=633 \mathrm{~nm}$ and for different polarization directions, indicated by the red arrow. The maps have been acquired by scanning the laser spot over the sample with steps of $200 \mathrm{~nm}$. The reflected signal is maximal for polarization direction parallel to the array axis and minimal for polarization perpendicular to the array axis. This effect is not observed for $\lambda_{L}=532 \mathrm{~nm}$, where no plasmonic resonance is excited [29]. The reflectance is normalized to that of the substrate.
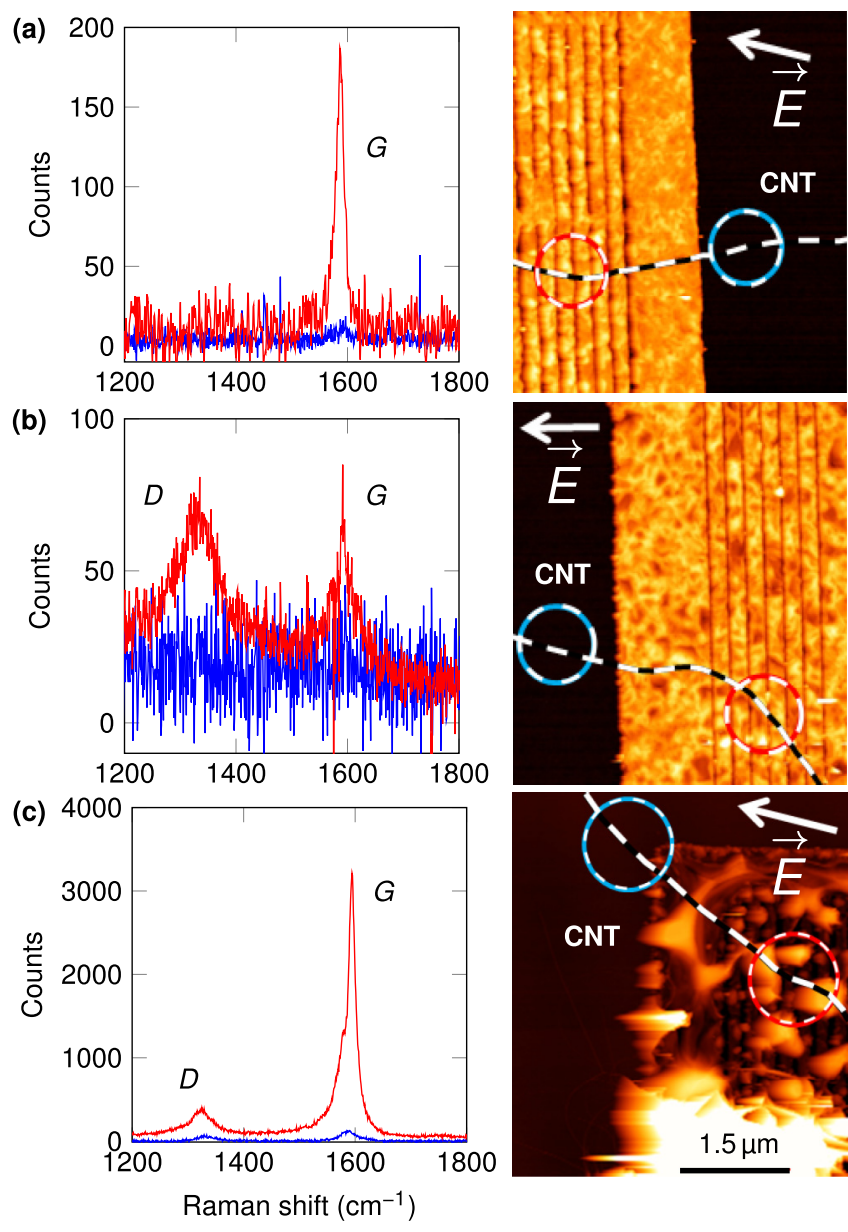

FIG. 3. Raman spectra $\left(\lambda_{L}=633 \mathrm{~nm}\right)$ for CNTs overgrown on optical antenna arrays (left panels) and corresponding AFM topography images (right panels). Red and blue curves refer to measurements on CNT portions overgrown on antennas or lying on the substrate, respectively. The red and blue circles indicate the position and the size of the focused light spot for the corresponding measurement. The white arrows indicate the polarization directions. In (a) and (b) the antenna strips consist of a Re/Pt bilayer. In (c) antenna strips consist of Ti/Pt. Compared to the structures above, the corrugations due to the CVD process are much more pronounced. All the Raman spectra have been obtained after $30 \mathrm{~s}$ of integration, except the bare CNT signal in (a), where the time was 10 times longer and the signal was then divided by a factor 10 .
The red curves on the left panels refer to the Raman signal around the $G$ and the $D$ peak measured on CNT segments located on the antenna strips, while the blue ones correspond to Raman measurements on bare portions. The spectra have been acquired after an integration time of $30 \mathrm{~s}$. The right panels in Fig. 3 show AFM topography scans of the three samples, where the red and light blue circles indicate the laser spot size and location for the bare CNT portion and for the CNT on antennas, respectively. The arrows indicate the incident light polarization for each measurement. The difference between red and blue curves in Figs. 3(a)-3(c) clearly shows the dramatic amplification of the Raman signal induced by the optical antennas.

In order to quantify the signal amplification, we define the enhancement factor as the ratio between the Raman peak heights measured with and without antennas. The enhancement factor is difficult to estimate when the signal on the bare portion of the CNT is too weak. The intensity of the Raman signal measured on a bare CNT depends on the difference between the incident $\hbar \omega_{L}$ (or the scattered $\hbar \omega_{L} \pm \hbar \omega_{q}$ ) photon energy and the energy separation $E_{i i}$ between VHSs. The width of such resonance windows is roughly of the order of $200 \mathrm{meV}$ for the $G$ mode and around $50 \mathrm{meV}$ for the radial breathing mode (RBM) [27,31]. If the difference between incident (or scattered) photon and the transition energy $E_{i i}$ is larger than the above values, then the Raman signal is drastically suppressed. This is often the case: for a given laser frequency $\omega_{L}$, only a small fraction of the CNTs shows a significant signal. This is precisely where the advantage of optical antennas becomes obvious, as the signal amplification compensates for the suppression due to the energy mismatch [22]. As demonstrated in Fig. 3, also CNTs which are scarcely or not measurable without antennas provide a significant signal. From the spectrum in Fig. 3(a) we deduce an enhancement factor of around 40 . While the signal on the bare portion of this CNT is barely discernible, the amplified signal provides useful information: the CNT has a low number of defects, as deduced by the absence of the $D$ peak; furthermore the narrow (FWHM: $17 \mathrm{~cm}^{-1}$ ) $G$ peak implies a very weak $G^{-}$component, which indicates either an achiral CNT (armchair or zigzag) or a CNT with very low chiral angle [32,33].

The graph in Fig. 3(b) refers to a CNT whose Raman spectrum is not discernible on the bare portion. This indicates that the CNT has no $E_{i i}$ transitions near $633 \mathrm{~nm}$. However, the signal on the CNT portion suspended on the antennas is 
clearly detectable, though weak. Since the $D$ peak is stronger than the $G$ peak, we deduce that this CNT has a relatively high number of defects.

Finally, Fig. 3(c) shows the results of Raman measurements on antennas made of Ti/Pt, i.e., without the Re sticking layer. As mentioned above, the absence of the Re sticking layer induce large corrugations in the Pt film when subjected to growth temperatures of the order of $850^{\circ} \mathrm{C}$. We note that despite the surface roughness the enhancement factor remains considerably high, of the order of 60. This particular CNT has a separation $E_{i i}$ between VHSs relatively close to the incident photon energy, thus the Raman signal is large enough to be detected without antennas as well. However, the antenna array allows us to measure also the RBM (the low-energy Raman spectrum for this CNT is shown in the Supplemental Material [29]). By following the standard assignment procedure we deduce the possible chiral indices for the CNT. We found that the most likely chiral indices are $(22,5)$ or $(21,7)$, two adjacent elements of the CNT family $2 n+m=49$ which share very similar properties, as, e.g., diameter, chiral angle, and energy separation between VHSs [29,34].

\section{DISCUSSION}

The widths of the plasmonic resonances measured in our reflectance spectroscopy experiments are of the order of $\Delta \lambda \approx 100 \mathrm{~nm}$. As shown below, simple model calculations considering metallic strips alone lead to plasmonic resonance widths much larger than the observed ones. One possibility to explain the observed narrow resonances is to consider in addition the optical interference due to the $\mathrm{SiO}_{2} / \mathrm{Si}$ interface $470 \mathrm{~nm}$ below the sample surface. We will show that the interplay between the optical mode in the $\mathrm{SiO}_{2}$ cavity and the plasmonic mode within the antenna strips gives rise to much sharper eigenmodes. Numerical simulations, discussed at the end of this section, show that such modes have a width comparable to that observed in the experiment.

In our analytical calculation [29] we approximate a single strip as an infinitely long cylinder of elliptical cross section. As sketched in Fig. 4(a) the field is evaluated in the position $Q$ located $5 \mathrm{~nm}$ away from the strip edge. The vertical axis $t$ measures $25 \mathrm{~nm}$ (equal to our antenna thickness) and the horizontal axis $w$ is varied. The graph in Fig. 4(b) shows the results of the calculation for the electric field amplitude (normalized to the incident field amplitude) as a function of both $w$ and the incident frequency $v$. We notice that, owing to the large imaginary part of the refractive index of Pt [35], for all widths $w$ the LSPR resonance width is much broader than that observed in our experiment.

In principle, the plasmonic eigenmodes of an array of strips differ from those of a single structure owing to the electrostatic interaction between the strips which leads to mode hybridization. However, such a difference is relevant only if the decay length for the near field amplitude in the vicinity of a strip is comparable to the gap between two adjacent strips. In the visible range such decay length for submicrometric plasmonic structures is of the order of a few nanometers [1,29], which is much smaller than the gap $(g=40 \mathrm{~nm})$. Therefore the interaction between adjacent strips can be neglected in our case.
The sharp minima observed in our reflectance spectroscopy experiment can be explained by considering the effect of the substrate. Indeed, even in a plain $\mathrm{SiO}_{2} / \mathrm{Si}$ substrate the electric field amplitude at the sample surface strongly depends on the frequency $v$, owing to the optical interference between the two surfaces of the $\mathrm{SiO}_{2}$ film. In Fig. 4(d) we plot the calculated electric field amplitude in a point located on the upper $\mathrm{SiO}_{2}$ surface. For ease of comparison with one of the following figures, the graph is plotted as a function of $w$ and $v$ as in Fig. 4(b), although in absence of nanoantennas the variable $w$ plays no role. The graph shows that the field amplitude at the surface considerably oscillates as a function of the frequency. Clearly this significantly alters the LSPR profile of optical antennas patterned on top of $\mathrm{a} \mathrm{SiO}_{2}$ film.

To quantify the impact of the substrate on the LSPR, we performed a finite-difference frequency-domain (FDFD) numerical simulation on the actual geometry, sketched in Fig. 4(e). In the calculation we model the nanoantennas as periodic arrays of infinitely long strips. By sweeping geometry parameters and laser frequency, we do not only extract field enhancement factors but also identify plasmonic modes and the interplay between the metal structure and the $\mathrm{SiO}_{2} / \mathrm{Si}$ substrate stack. The strip section is assumed to be rectangular with rounded corners (with radius of curvature $r=5 \mathrm{~nm}$ ). The gap $g=40 \mathrm{~nm}$, the etching depth $e=100 \mathrm{~nm}$, and the $\mathrm{SiO}_{2}$ thickness $s=470 \mathrm{~nm}$ are kept constant. The result of the simulation is plotted in Fig. 4(f). The graph shows the horizontal component of the electric field amplitude calculated in the point $Q$ indicated in the sketch, plotted as a function of width $w$ and frequency $v$. A comparison with the graphs in Figs. 4(b) and 4(d) reveals that the actual eigenmodes are nontrivial combinations of the cavity modes in the $\mathrm{SiO}_{2}$ layer and the plasmonic modes in the metal strip. In fact, the graph in Fig. 4(f) shows a broad feature (on the left and bottom part of the graph) modulated by almost vertical fringes which are clearly related to the $\mathrm{SiO}_{2}$ film interference. As a result, in the visible range the maximum of the near field amplitude for a 200-nm-wide strip occurs at approximately $470 \mathrm{THz}$, i.e., close to the red laser frequency. We also notice several arc-shaped features on the top-right zone of the graph, which correspond to higher energy modes. Further details about the calculation and the interpretation of optical modes are given in the Supplemental Material [29].

The reflection coefficient for a thin film deposited on top of a high refractive index substrate displays a maximum when the electric field at the surface is minimal [29]. This condition corresponds to a frequency $v$ such that the electric field forms a standing wave within the $\mathrm{SiO}_{2}$ layer, with nodes at the two interfaces (constructive interference). Vice versa, when the reflection coefficient is minimal (destructive interference), the electric field at the surface is maximal. On the other hand, when the polarizability $\alpha$ of an optical antenna (and thus the near field amplitude) has a maximum as a function of $v$ then, due to the imaginary part of the metal refractive index, the reflected far field shows a minimum. In order to obtain the largest field amplification by thin plasmonic structures, it is necessary that the electric field at the surface is maximal. This corresponds to the case of destructive interference [36] for the far-field reflection by a thin dielectric. Therefore, our definition 
(a)

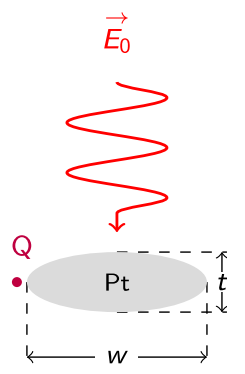

(b)

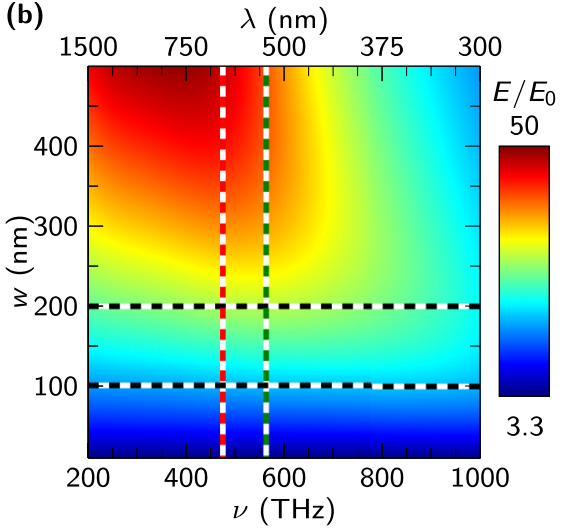

(c)

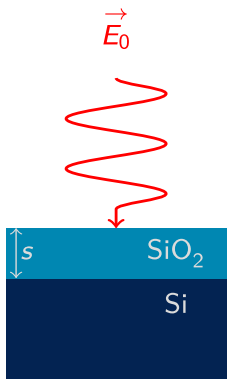

(d)

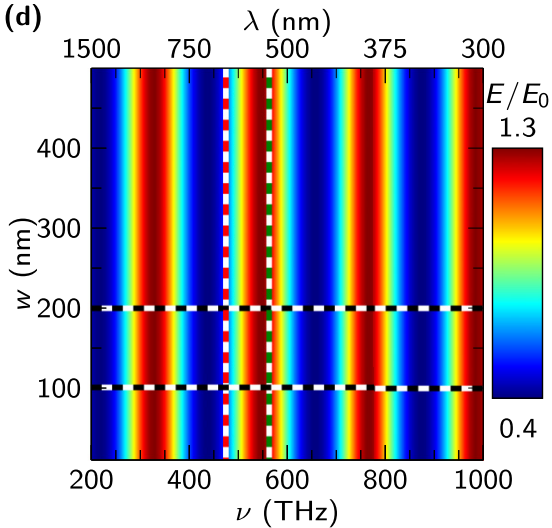

(g)

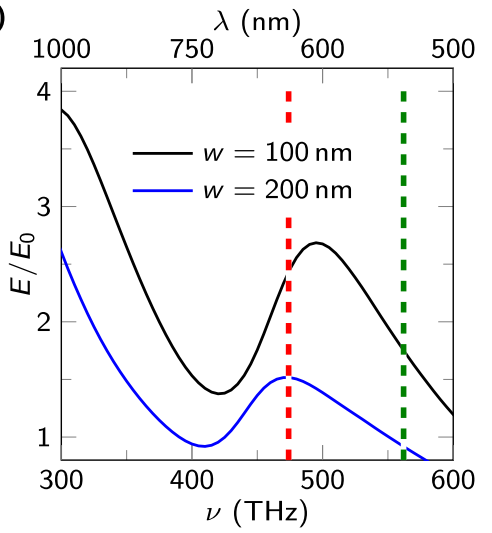

FIG. 4. (a) Sketch of the geometry considered in the analytical calculations plotted in (b). An infinitely long Pt strip of elliptical section is illuminated from above by a plane wave of amplitude $\left|E_{0}\right|$. The incident field polarization is perpendicular to the strip axis. The vertical axis $t=25 \mathrm{~nm}$ equals the thickness of the strips in our experiment. The point $Q$ indicates the position where the field strength is calculated. (b) Corresponding color plot of the electric field intensity calculated in the point $Q$ at $5 \mathrm{~nm}$ from the strip edge. The field is plotted as a function of strip width $w$ and excitation frequency $v$. (c) In a complementary calculation we consider the effect of the substrate alone (see text). The substrate consists of a doped $\mathrm{Si}$ wafer capped with a 470-nm-thick $\mathrm{SiO}_{2}$ layer. (d) The corresponding color plot for the electric field amplitude at the top surface of the $\mathrm{SiO}_{2}$ layer. (e) Sketch of the geometry used in our finite-difference frequency-domain (FDFD) simulation. We used the following parameters: gap $g=40 \mathrm{~nm}$, etching depth $e=100 \mathrm{~nm}, \mathrm{SiO}_{2}$ layer thickness $s=470 \mathrm{~nm}$, strip thickness $t=25 \mathrm{~nm}$ (the Re and Pt layers are 7 and $18 \mathrm{~nm}$ thick, respectively) The simulation box width and height are $b=240 \mathrm{~nm}$ and $h=1000 \mathrm{~nm}$, respectively. The corners of the metal strip have been rounded (curvature radius $r=5 \mathrm{~nm}$ ). The width $w$ is swept from 10 to $500 \mathrm{~nm}$ and the field frequency $v$ is swept from 200 to $1000 \mathrm{THz}$. (f) Electric field amplitude calculated by FDFD in the point $Q$ located along the strip axis at $5 \mathrm{~nm}$ from the metal edge. (g) Graph of the electric field amplitude as a function of the incident frequency for $w=100$ and $200 \mathrm{~nm}$, corresponding to the two horizontal cuts in (f).

of enhancement factor is, strictly speaking, not absolute but relative to the substrate.

A conclusion drawn from the calculations above is that the dependence of the antenna resonance frequency on both antenna width and antenna gap is relatively smooth. Therefore the roughness produced by the CVD process has a negligible effect on both the resonance frequency and the enhancement factor. This can explain the good enhancement factor observed in Fig. 3(c) despite the surface roughness of the metal stripes of that sample.

\section{CONCLUSIONS}

We have demonstrated directional optical antennas for applications that require extremely high process temperatures as, e.g., those required for the CVD growth of CNTs. We have fabricated devices where CNTs are grown on top of antenna arrays and shown that the latter significantly enhance the Raman response of the CNT by factors ranging from 3 to 40 . Numerical simulations show that the relatively sharp antenna resonance is due to the interplay of plasmonic resonance and thin film interference due to the $\mathrm{SiO}_{2}$ cap layer. Possible applications of Pt-Re optical antennas go well beyond SERS of CNTs, since the present fabrication scheme can be in principle applied to any nanostructure for optical spectroscopy whose fabrication requires extreme temperature conditions.

\section{ACKNOWLEDGMENTS}

The work was funded by the European Union within the STREP SE2ND, and by the Deutsche Forschungsgemeinschaft within Grants No. SFB689 and No. GRK1570. 
[1] L. Novotny and B. Hecht, Principles of Nano-Optics (Cambridge University Press, Cambridge, 2006).

[2] M. S. Tame, K. R. McEnery, S. K. Özdemir, J. Lee, S. A. Maier, and M. S. Kim, Nat. Phys. 9, 329 (2013).

[3] K. Kneipp, Y. Wang, H. Kneipp, L. T. Perelman, I. Itzkan, R. R. Dasari, and M. S. Feld, Phys. Rev. Lett. 78, 1667 (1997).

[4] S. Nie, Science 275, 1102 (1997).

[5] R. Chikkaraddy, B. de Nijs, F. Benz, S. J. Barrow, O. A. Scherman, E. Rosta, A. Demetriadou, P. Fox, O. Hess, and J. J. Baumberg, Nature (London) 535, 127 (2016).

[6] M. Moskovits, Rev. Mod. Phys. 57, 783 (1985).

[7] R. Stosch, F. Yaghobian, T. Weimann, R. J. C. Brown, M. J. T. Milton, and B. Güttler, Nanotechnology 22, 105303 (2011).

[8] K. Kneipp, H. Kneipp, M. S. Dresselhaus, and S. Lefrant, Philos. Trans. R. Soc. London Sect. A 362, 2361 (2004).

[9] M. Fleischmann, P. J. Hendra, and A. J. McQuillan, Chem. Phys. Lett. 26, 163 (1974).

[10] J. A. Creighton, in Surface Enhanced Raman Scattering, edited by R. K. Chang and T. E. Furtak (Plenum, New York, 1982), p. 315 .

[11] R. Benner, K. V. Raben, K. Lee, J. Owen, R. Chang, and B. Laude, Chem. Phys. Lett. 96, 65 (1983).

[12] D. A. Weitz and M. Oliveria, Phys. Rev. Lett. 52, 1433 (1984).

[13] P. N. Sanda, J. M. Warlaumont, J. E. Demuth, J. C. Tsang, K. Christmann, and J. A. Bradley, Phys. Rev. Lett. 45, 1519 (1980).

[14] P. Liao, J. Bergman, D. Chemla, A. Wokaun, J. Melngailis, A. Hawryluk, and N. Economou, Chem. Phys. Lett. 82, 355 (1981).

[15] In the literature CNTs which have never been subjected to any fabrication step and are not interacting with the substrate are called ultraclean CNTs.

[16] J. Cao, Q. Wang, and H. Dai, Nat. Mater. 4, 745 (2005).

[17] E. A. Laird, F. Kuemmeth, G. A. Steele, K. Grove-Rasmussen, J. Nygård, K. Flensberg, and L. P. Kouwenhoven, Rev. Mod. Phys. 87, 703 (2015).

[18] R. Saito, G. Dresselhaus, and M. S. Dresselhaus, Physical Properties of Carbon Nanotubes (Imperial College Press, London, 1998).

[19] K. Kneipp, H. Kneipp, P. Corio, S. D. M. Brown, K. Shafer, J. Motz, L. T. Perelman, E. B. Hanlon, A. Marucci, G. Dresselhaus, and M. S. Dresselhaus, Phys. Rev. Lett. 84, 3470 (2000).

[20] H. Chu, J. Wang, L. Ding, D. Yuan, Y. Zhang, J. Liu, and Y. Li, J. Am. Chem. Soc. 131, 14310 (2009).

[21] S. Heeg, A. Oikonomou, R. Fernandez-Garcia, C. Lehmann, S. A. Maier, A. Vijayaraghavan, and S. Reich, Nano Lett. 14, 1762 (2014).

[22] N. Paradiso, F. Yaghobian, C. Lange, T. Korn, C. Schüller, R. Huber, and C. Strunk, Phys. Rev. B 91, 235449 (2015).

[23] J. Cao, Q. Wang, M. Rolandi, and H. Dai, Phys. Rev. Lett. 93, 216803 (2004).

[24] A. Dirnaichner, M. del Valle, K. J. G. Götz, F. J. Schupp, N. Paradiso, M. Grifoni, C. Strunk, and A. K. Hüttel, Phys. Rev. Lett. 117, 166804 (2016).
[25] H. Kataura, Y. Kumazawa, N. Kojima, Y. Y. Maniwa, I. Umezu, S. Masubuchi, S. Kazama, X. Zhao, Y. Ando, Y. Ohtsuka, S. Suzuki, and Y. Achiba, Proceedings of the IWEPNM'99 (American Institute of Physics, Woodbury, NY, 1999).

[26] A. Jorio, R. Saito, J. H. Hafner, C. M. Lieber, M. Hunter, T. McClure, G. Dresselhaus, and M. S. Dresselhaus, Phys. Rev. Lett. 86, 1118 (2001).

[27] J. Maultzsch, H. Telg, S. Reich, and C. Thomsen, Phys. Rev. B 72, 205438 (2005).

[28] L. Le Thi Ngoc, M. Jin, J. Wiedemair, A. van den Berg, and E. T. Carlen, ACS Nano 7, 5223 (2013).

[29] See Supplemental Material at http://link.aps.org/supplemental/ 10.1103/PhysRevB.96.035408 for further details. The Supplemental Material includes Refs. [37-46].

[30] S. Huang, X. Cai, and J. Liu, J. Am. Chem. Soc. 125, 5636 (2003).

[31] M. Fouquet, H. Telg, J. Maultzsch, Y. Wu, B. Chandra, J. Hone, T. F. Heinz, and C. Thomsen, Phys. Rev. Lett. 102, 075501 (2009).

[32] R. Saito, A. Jorio, J. H. Hafner, C. M. Lieber, M. Hunter, T. McClure, G. Dresselhaus, and M. S. Dresselhaus, Phys. Rev. B 64, 085312 (2001).

[33] J. S. Park, K. Sasaki, R. Saito, W. Izumida, M. Kalbac, H. Farhat, G. Dresselhaus, and M. S. Dresselhaus, Phys. Rev. B 80, 081402 (2009).

[34] K. Liu, J. Deslippe, F. Xiao, R. B. Capaz, X. Hong, S. Aloni, A. Zettl, W. Wang, X. Bai, S. G. Louie, E. Wang, and F. Wang, Nat. Nanotechnol. 7, 325 (2012).

[35] A. D. Rakić, A. B. Djurišić, J. M. Elazar, and M. L. Majewski, Appl. Opt. 37, 5271 (1998).

[36] D. Yoon, H. Moon, Y.-W. Son, J. S. Choi, B. H. Park, Y. H. Cha, Y. D. Kim, and H. Cheong, Phys. Rev. B 80, 125422 (2009).

[37] C. F. Bohren and D. R. Hoffman, Absorption and Scattering of Light by Small Particles (Wiley-Interscience, NewYork, 1983).

[38] M. Meier and A. Wokaun, Opt. Lett. 8, 581 (1983).

[39] A. Wokaun, J. P. Gordon, and P. F. Liao, Phys. Rev. Lett. 48, 957 (1982).

[40] A. Wokaun, in Solid State Physics, edited by H. Ehrenreich, F. Seitz, and D. Turnbull (Academic, New York, 1984), Vol. 38.

[41] O. Stenzel, The Physics of Thin Film Optical Spectra, An Introduction (Springer, Berlin, 2005).

[42] R. Saito, M. Hoffman, G. Dresselhaus, A. Jorio, and M. S. Dresselhaus, Adv. Phys. 60, 413 (2011).

[43] S. Adachi, The Handbook on Optical Constants of Metals (World Scientific, Singapore, 2012).

[44] M. A. Green and M. J. Keevers, Progress Photovolt. Res. Appl. 3, 189 (1995)

[45] I. H. Malitson, J. Opt. Soc. Am. 55, 1205 (1965).

[46] Y. Todorov, A. M. Andrews, I. Sagnes, R. Colombelli, P. Klang, G. Strasser, and C. Sirtori, Phys. Rev. Lett. 102, 186402 (2009). 